INNOVATIONS IN PRIMARY CARE

\title{
An In-Clinic Food Pharmacy Addresses Very Low Food Security
}

\author{
Emily Cooper, MD, MHA, \\ FAAFP' \\ Sveta Mobanan, $M D^{1}$ \\ Christina Garcia, DO, MPH \\ Tamara Baldwin Thomas, \\ MSW, LCSW'
}

Ann Fam Med 2020;18:564. https://doi.org/10.1370/afm.2603.

\section{INNOVATION}

Food insecurity is consistently associated with negative health outcomes, ${ }^{1}$ and health systems are increasingly interested in addressing the social determinants of health, including food access, within the clinical space. Collaborating with a local food pantry partner, we developed a food pharmacy that provided a 2-day supply of balanced, healthy, shelf-stable foods, embedded into an outpatient primary care clinic, to address very low food security among patients. ${ }^{2}$

\section{WHO \& WHERE}

Atrium Health's Biddle Point Family Medicine Clinic and Community Health division partnered with Loaves \& Fishes, a local network of food pantries, to establish the food pharmacy at the clinic, which historically serves medically and socially complex patients of lower socioeconomic status in Charlotte, North Carolina. In 2016, Atrium Health implemented a Community Health Improvement Study that identified specific neighborhoods as food deserts and Loaves $\&$ Fishes implemented a mapping process to show where their clients were coming from. These maps showed overlaps and a shared opportunity to increase access to food. Additionally, dietitians from Atrium Health partnered with clinicians at Biddle Point and Loaves $\&$ Fishes to select healthier shelf-stable options for the food pharmacy (ie, low-sodium, lowsugar, whole grain, etc) and developed educational materials. Loaves $\&$ Fishes delivers the food monthly to the clinic.

\section{HOW}

For a month-long pilot, 3 physicians screened adult, Englishspeaking patients using 15 questions from the Hunger Vital

Conflicts of interest: autbors report none.

\section{Corresponding author}

Brisa Hernandez

9477 Caddell Rd

Fort Mill, SC 29707

Brisahernandez07@gmail.com
Signs and the Protocol for Responding to and Assessing a Patient's Assets, Risks, and Experiences (PRAPARE) tool. Patients were given a laminated screening form at checkin. The rooming nurse collected the form and entered the responses into the patient's electronic medical record (EMR); the physician reviewed the patient's responses before entering the exam room.

If patients reported experiencing food insecurity, clinicians offered a referral to the in-clinic food pharmacy for a 2-day emergency supply of food (Supplemental Figure 1), as well as a referral to the in-house social worker to discuss longer-term solutions to food insecurity (including referrals to Loaves and Fishes pantries). Physicians designated a food pharmacy prescription as a Miscellaneous Medication. At check-out, patients would exchange the paper prescription for a bag of food from the food pharmacy. Recognizing the patient is likely not the lone person in the household, additional resources were provided that would address the need of others in their household.

After the pilot, we expanded screening to include all adult English- and Spanish-speaking patients at yearly and new patient visits. All clinicians were eligible to refer patients to the food pharmacy. Patient education was developed about why these screening questions are being implemented in the clinical setting and encouraging conversation with their clinicians.

\section{LEARNING}

Screening for social needs identified the importance of using a short, low-reading-level screener, translated into multiple languages, with an identical and simple EMR data collection tool. The food pharmacy was helpful for patients currently experiencing very low food security; however, several patients described the cyclical and sometimes predictable nature of food insecurity. Patients also expressed difficulty acquiring fresh fruits and vegetables, which suggests a need for increased access to fresh foods. Clinicians and staff also recognized that it was important to respond to the needs of patients beyond just providing referrals to resources. It was also clear that adding this type of information to the visit also changes the dynamic of the patient-clinician relationship. Ongoing discussions need to happen in order to support both patients and staff as this type of screening and support is provided.

For additional information, including affiliations, figure, key words, funding support, acknowledgments, and references, see https://www.AnnFamMed.org/content/18/6/564/suppl/DC1/.

Submitted June 20, 2019; submitted, revised, April 7, 2020; accepted April 16, 2020. 